\title{
Julien Godest, paysan autobiographe sous l'aile de Taldir-Jaffrennou
}

Julien Godest, a peasant autobiographer who was taken under the wing of Taldir-Jaffrennou

\section{Nelly Blanchard}

\section{OpenEdition}

Journals

Édition électronique

URL : https://journals.openedition.org/lbl/986

DOI : $10.4000 / \mathrm{lbl} .986$

ISSN : 2727-9383

\section{Éditeur}

Université de Bretagne Occidentale - UBO

\section{Édition imprimée}

Date de publication : 1 mars 2014

Pagination : 7-31

ISBN : 979-10-92331-07-3

ISSN : $1270-2412$

\section{Référence électronique}

Nelly Blanchard, « Julien Godest, paysan autobiographe sous l'aile de Taldir-Jaffrennou », La Bretagne Linguistique [En ligne], 18 | 2014, mis en ligne le 01 mai 2021, consulté le 21 mai 2021. URL : http:// journals.openedition.org//bl/986 ; DOl : https://doi.org/10.4000/lbl.986

\section{(c) (1)}

La Bretagne Linguistique est mise à disposition selon les termes de la Licence Creative Commons Attribution 4.0 International. 


\section{Julien Godest, paysan autobiographe sous l'aile de Taldir-Jaffrennou}

\footnotetext{
$\mathrm{J}_{\mathrm{s}}$
} e propose de me pencher sur un nouvel exemple de littérature «populaire», populaire dans le sens où elle est le fruit de la plume d'un homme du peuple, d'un paysan. Après avoir exploré l'univers du paysan de Plouider Hervé Burel, je me plonge dans celui, fort différent, de Julien Godest, «Juluen al laezh» ou «Juluen al laezh dous», comme on l'appelait dans sa région - car il vendait du lait tous les jours à Callac et dans les environs. Julien Godest a en effet écrit, tout comme Burel, une autobiographie à laquelle il a donné le titre de Envorennou ar barz Juluen Godest (Les Souvenirs du barde Julien Godest), qu'il a sans doute rédigée vers 1910-14, et qui se trouve actuellement dans le fonds Jaffrennou des Archives départementales du Finistère (Quimper). D'une belle écriture, il a, lui aussi, noirci quelques centaines de pages (environ 300) pour laisser une trace et surtout une image de sa vie.

Par contre, à la différence d'Hervé Burel, le texte de Julien Godest a partiellement été édité, environ 10-13\% du manuscrit. C'est François Jaffrennou, homme connu du mouvement breton, qui

\footnotetext{
* Maître de conférences de breton, CRBC (EA 4451-UMS 3554), UBO/ueb.
} 
en a pris l'initiative. En 1929 il décide de publier dans sa revue $A n$ Oaled «Buez eur Paour kez Kouer, gant Juluen Godest», autrement dit la «Vie d'un pauvre paysan, par Julien Godest». Quatre numéros de la revue ouvrent ainsi la porte à la plume de cet homme et font de Julien Godest un auteur. Jaffrennou a en effet estimé que Julien Godest était un écrivain et un écrivain qui méritait d'être lu. Toutefois, non seulement le manuscrit n'a pas été publié entièrement puisque Jaffrennou a fait des choix d'extraits, mais une comparaison entre le manuscrit et le texte publié met en lumière un assez grand nombre d'interventions de l'éditeur sur le texte de l'auteur.

Cette rencontre entre un éditeur et un auteur, mais surtout entre un homme de l'Emsao et un homme du peuple, m'a paru être un angle d'approche intéressant pour essayer de comprendre la vision que la nouvelle génération de l'Emsao, plutôt bourgeoise en ce début de $\mathrm{XX}^{\mathrm{e}}$ siècle, a du peuple. Comment comprendre cette apparente contradiction : pourquoi Jaffrennou a-t-il considéré Godest comme un auteur devant absolument être lu, mais comme un auteur illisible en l'état?

\section{La rencontre Godest-Jaffrennou : le peuple écrivain}

\section{Le peuple-lecteur et le peuple-source chez Taldir-Jaffrennou}

François Jaffrennou, né à Carnoët en 1879 (mort à Bergerac en 1956), fils de notaire, a fait des études à Guingamp au collège de Notre Dame de Bon Secours puis à Saint-Brieuc au collège Saint-Charles. Il y a reçu des enseignements de deux personnalités qui ont marqué le mouvement breton : l'abbé Le Clerc et François Vallée. Il s'adonne d'abord au journalisme, puis se lance lui-même dans l'édition de journaux : Ar Vro (Le Pays, 1904-1914, tout en breton), et $\mathrm{Ar} \mathrm{Bobl}$ (Le Peuple, 1904-1914, bilingue). Entre les deux guerres, il dirige deux périodiques bilingues : Le Consortium breton (1927-28) et An Oaled/Foyer breton (1928-1939). Il s'est engagé très jeune dans le mouvement breton et y a joué un rôle d'initiateur, d'organisateur, de stimulateur : il a co-fondé l'Union Régionaliste Bretonne en 1898, a pris le nom de barde Taldir à l'Eiseddfod de Cardiff en 1899 et tissé des liens entre l'Emsao et les autres pays celtiques, a créé l'Association des Étudiants Bretons à Rennes et le Ti Kaniri Breiz en 1900. Il intègre le Gorsedd des Bardes et Druides de Petite-Bretagne 
et devient Grand Druide en 1933. Il est également connu comme auteur de l'hymne national breton «Bro Goz ma Zadou».

On sent à la fois chez lui, comme chez toute la jeune génération des personnes faisant de la Bretagne et du breton des objets dignes d'intérêt et de revendication, une filiation revendiquée avec les prédécesseurs bretonistes, d'origine noble le plus souvent, mais également leur mise à l'écart respectueuse, mais de plus en plus nette, mise à l'écart qui petit-à-petit fera passer l'Emsao du régionalisme au nationalisme. Dans le premier numéro d'Ar Bobl (24 septembre 1904), Jaffrennou publie un article lui permettant d'expliquer ce qu'est le journal : «Petra omp ?» Il y affirme clairement ses objectifs :

"Unvaniez hag adsao; ia, setu eno petra neuz komanset ar rejionalisted ober en Breiz-Izel, ha mar n'int ket deut c'hoaz abenn eus o zaol, mar n'o deuz ket graet c'hoaz gant hon bro eur vro unanet stard evid difenn he interest he-unan, he c'hiziou leun a vraventez, he c'harakter hag he langach, da vihana an hent a zo bet merket ganto ha dre an hent-se e kerzo ive gant kalon hon c'hazeten neve c'hanet.» (Union et relèvement; oui, voici ce que les régionalistes ont commencé à faire en Basse-Bretagne, et s'ils n'ont pas encore touché leur but, s'ils n'ont pas encore fait de notre pays un pays fortement uni pour défendre ses propres intérêts, ses mœurs pleins de beauté, son caractère et sa langue, ils ont au moins tracé le sillon et c'est aussi ce chemin qu'empruntera avec courage notre journal nouvellement né.)

Puis il rebondit sur l'idée d'unité en ne mentionnant certes pas la distance qui sépare les nobles du peuple, mais en insistant sur la proximité de la classe bourgeoise avec le peuple, faisant de celle-ci la classe qui doit prendre le relais de la conscientisation bretonne : "Ar baizanted hag an ouvrierien vreton a gavo ennomp ama ouspenn kenvroïz, ouspen harperien : mignoned d'ezo e vemp c'hoaz, mignoned bet maget ha savet en o mesk, a oar petra eo o ezommou» (Les paysans et les ouviers bretons reconnaitront en nous, non seulement des compatriotes, non seulement des soutiens : nous serons leurs amis, des amis nourris et élevés parmi eux et qui savent ce que sont leurs besoins).

Cette redéfinition de la relation au peuple de la part de l'Emsao se caractérise par la prise de conscience de la capacité du peuple à recevoir de la nourriture intellectuelle et par une prise en compte 
de la valeur des productions émanant du peuple. La relation élite intellectuelle-peuple doit cesser de se faire à sens unique - l'élite collecte le trésor littéraire oral du peuple, mais ne lui apporte rien d'autre que la charité -, elle doit se baser désormais sur un échange intellectuel, d'où la création d'un grand nombre de journaux ou périodiques en langue bretonne ou bilingues au tournant des XIX ${ }^{\mathrm{e}}$ et $\mathrm{XX}^{\mathrm{e}}$ siècles. Le titre, $A r B o b l$, choisi par Jaffrennou pour sa production n'est évidemment pas anodin, il témoigne de cet élan populiste, de cette ambition de production d'une nourriture intellectuelle pour le peuple : "an hano Ar Bobl gant pehini eo bet badezet ar journalma, n'eo ket eun hano ven ha dilavar. An hano ze a zinifi penaoz eo d'ar bobl dresit-holl eo e vo gouestlet ar gazeten-ma» (Le nom Le Peuple par lequel nous avons baptisé ce journal n'est pas un nom vain et creux. Ce nom signifie que c'est avant tout au peuple que sera destiné ce journal). Le choix de la devise du journal renforce cette optique : «evid ar Vro dre ar Bobl : evid Breiz dre ar Vreiziz» (pour le Pays par le Peuple : pour la Bretagne par les Bretons). C'est la même motivation qui anime Jaffrennou lors de la création du Ti Kaniri Breiz : aller à la rencontre du peuple, de commune en commune, chanter sur la place publique, ouvrir les oreilles et les esprits. L'édition de feuilles volantes, comme Soniou Taldir evid ar bobl (Chansons de Taldir pour le peuple), relève aussi du même objectif : pour deux sous, offrir des chansons en langue bretonne et de courts articles sur la Bretagne ou la langue bretonne.

Le peuple devient donc clairement lecteur dans l'esprit des nouveaux dirigeants de l'Emsao, mais il devient aussi source littéraire. Il était déjà perçu auparavant comme source littéraire, toutefois, on ne se contente désormais plus, à la manière des premiers collecteurs du $\mathrm{XIX}^{\mathrm{e}}$ siècle, de considérer que le peuple est dépositaire d'un trésor concentré dans la littérature orale (chants, contes, etc.) et dont il se fait le porteur sans le savoir ; on commence au contraire à penser qu'il peut également produire des œuvres nouvelles, originales, personnelles. Jaffrennou semble ainsi s'être mis à la recherche d'hommes capables de se mettre à l'écriture et pouvant montrer que du peuple peut jaillir une littérature bretonne digne de ce nom. C'est ainsi qu'il a encouragé un maréchal-ferrant de Bodilis, Laurent Moysan ${ }^{1}$, à se

1. Laurent Moysan, né à Plougar en 1861, a fait son service vers 1885, marié à Angèle 
mettre à composer des chansons. Dix-sept chansons de sa plume ont été retrouvées et l'une d'entre elles se prolonge par une note explicative sur son activité de barde : après avoir expliqué qu'il avait commencé à écrire lors de son service militaire, vers 25 ans, il indique que "abaoe e oant chomet kousket. A dra zur ne vichent ket bet dihuned ma ne vije deuet "Taldir» gant e c'hourinadegou. Ne meuz ket a zeskadurez, med c'hoant e meuz da zikour va c'henvroiz ag e rign keit a ma c'hallin. Gant ar fizianz e meuz e vezimp treac'h dar re o deuz c'hoant dhon diskar» (depuis elles étaient restées endormies. Et certainement qu'elles ne se seraient pas réveillées si Taldir n'était pas venu avec ses concours. Je n'ai pas d'instruction, mais je veux aider mes compatriotes et je le ferai tant que je le pourrai. Et je suis sûr que nous vaincrons ceux qui veulent nous réduire).

C'est ce même rôle de stimulateur et de révélateur qu'a joué Jaffrennou auprès de Julien Godest.

\section{Un paysan-barde trouve un bienfaiteur}

Julien Godest est bien plus âgé que Jaffrennou. Il est né à la Chapelle-Neuve le 2 mars 1849 et mort à Callac en 1932. Il s'est marié à 21 ans et a eu 10 enfants. Il est issu d'une famille de paysans pauvres, a exercé le métier de métayer et a fait des tournées de vente de lait à Callac. Il n'a jamais fréquenté l'école. Alors qu'il avait 35 ans, son fils lui a appris à lire et à écrire. Il s'est rendu maître de son destin, il avait tellement de choses à raconter qu'il s'est donné les moyens d'y parvenir. Et la rencontre avec Jaffrennou a sans aucun doute été un catalyseur dans l'aventure littéraire de cet homme.

Jaffrennou et Godest se sont rencontrés sur la place de Callac en 1904. C'est Godest qui s'est adressé à Jaffrennou en lui disant qu'il savait qui il était, même si Jaffrennou, lui, ne savait sans doute pas qui était son interlocuteur. Jaffrennou fut surpris de l'intérêt que Godest portait à son pays et à sa langue maternelle, il reconnut en lui un homme hors du commun, "na oa ket deuz niver al labourerien a lavar eo awalc'h d'ezo 'turc'hial o douar'» (il ne faisait pas partie des agriculteurs qui disent que cela leur suffit de retourner la terre); selon lui, Godest savait élever son âme et tourner son regard vers

Berthou en 1887, père de cinq enfants, mort en 1938. Cf. Bulletin communal de Bodiliz, présentation faite par Alain Le Verge («E korn ar vrezonegerien»). 
la beauté du temps passé. Et il attribuait cela au fait que ce paysan venait d'une famille si pauvre qu'il n'avait pas pu être scolarisé et que «biskoaz 'skol gallek' na labeaz e natur breton!» (jamais aucune école française n'avait sali sa nature bretonne). Jaffrennou y a même reconnu un barde à la manière des bardes celtes : "Juluan Godest a zo eur barz, gret war batrom ar re-goz. En e greiz, a-dreuz d'ar c'hantvejou, eo beo an hevelep tan hag a lake da zaillat evit o iez hag o bro kalonou barzed evel Gwic'hlan, Aneurin, ha war o lerc'h ar C'hloareien o deuz skrivet ar mysteriou, an nouelou, hag ar c'hanaouennou koz a lenner er Barzaz-Breiz ${ }^{2}$ » (Julien Godest est un barde, sur le modèle des anciens bardes. En lui, au travers des siècles, brûle le même feu qui faisait s'engager pour leur langue et leur pays les cœurs des bardes comme Gwic'hlan, Aneurin, et à leur suite les clercs qui ont écrit les mystères, les noëls et les vieilles chansons qu'on lit dans le Barzaz-Breiz).

Après avoir contacté son ami Théodore Botrel, ils décident tous les deux de publier imméditement quelques gwerz de Godest. Botrel écrit : «Tu dis vrai, mon cher Fanch! Je ne suis pas riche (malgré la vogue), mais ta lettre m'a vivement touché, et je te remercie de me signaler cette belle et bonne action à accomplir. Le Barde Godest est vieux, eh bien, au soir de sa vie, avant l'orage menaçant, aidons-le tous deux, nous qui sommes jeunes, à rentrer son blé, à engranger sa récolte...»

Entre temps, Godest s'est remis à l'écriture, sans doute encouragé, voire influencé, par Jaffrennou. Il a sans doute confié son long texte autobiographique à son bienfaiteur (il semble que Jaffrennou se le soit d'ailleurs approprié puisque le manuscrit contient un ex-libris au nom de Jaffrennou / Tal-dir) qui, en 1929, estime que Godest doit encore connaître la publication pour ce qu'il vient d'écrire et décide de publier des extraits de cette autobiographie dans An Oaled:

- tome $4, n^{\circ} 19$ à 21,1929, p. 623-634;

- tome $5, \mathrm{n}^{\circ} 26-27,1929$, p. $737-743$;

- tome $5, \mathrm{n}^{\circ} 28,1929$, p. $835-839$;

- tome $5, \mathrm{n}^{\circ} 30,1929$, p. 903-906.

2. Préface de Taldir-Jaffrennou dans Juluan Godest, Dastumaden Gwerziou poblus, Saint-Brieuc, Prud'homme, 1904 (48 p.). 


\section{Le traitement de l'autobiographie de Godest : l'illisibilité du peuple}

\section{Une édition partielle}

La publication de Godest dans An Oaled ne porte pas le même titre que celui choisi par l'auteur. Il n'y est plus question de barde : alors que Godest souhaitait parler des «Souvenirs du barde Julien Godest», Jaffrennou souhaite que soit lue la «Vie d'un pauvre paysan, par Julien Godest». La publication au sein d'une revue et non sous forme d'ouvrage ne permettait évidemment pas une publication in extenso du manuscrit et des choix et coupures s'imposaient. On note en effet que Jaffrennou ne s'est intéressé qu'à la première moitié du manuscrit, et ne s'est par exemple pas penché sur la partie du texte de Godest qui évoque justement sa vocation de barde : "Galvidiges bars Juluen Godest, da laret eo galvet dar varzoniez». Seuls les événements qui se sont succédé et ont construit sa vie semblent l'avoir intéressé.

De plus, même dans cette première grande partie sur la vie de Godest, des choix ont été faits. Jaffrennou a ordonné un texte publié en 16 parties, ce que Godest n'a pas fait :

1. Godest en em Drezant (sic) e-unan (Godest se présente luimême)

2. Godest o tiskleria pesort bue a rene e Dadou (Godest explique la vie menée par ses ancêtres)

3. Iaouankiz Juluen Godest (La jeunesse de Julien Godest)

4. Godest o vond da Zoudard evid 7 vla (Godest partant au service pour 7 ans)

5. Godest ebarz ar C'Hazern (Godest à la caserne)

6. Pesort hunvre a reaz Godest (Le rêve que fit Godest)

7. Petra a welaz Godest er Baradoz (Ce que Godest vit au paradis)

8. Ar brezonek a ve komzet er Baradoz (C'est le breton que l'on parle au paradis)

9. Godest hag ar C'horonel (Godest et le Colonel)

10. Micher soudard Godest (Le métier de soldat de Godest)

11. Penoz a varvaz Toutois, kamarad-gwele Godest (La mort de Toutois, camarade de chambre de Godest) 
12. Tokou mod Breiz-Izel d'ar soudarded (Des chapeaux à la mode de Bretagne aux soldats)

13. Brezel dek-ha-tri-ugent. - Ar Gommun (La guerre de 70 - La Commune)

14. Heuzusded ar Gommun (Les horreurs de la Commune)

15. Distro ar soudard d'ar ger (Le retour du soldat chez lui)

16. Godest o temezi (Godest se marie)

Enfin, il est important de noter que les parties 4 à 15 sont publiées comme s'il s'agissait de la vie de Godest, précisément l'époque du service militaire. Or Julien Godest n'a jamais fait 7 ans de service. C'est de son frère Jean-François-Marie Godest qu'il s'agit. Jaffrennou a-t-il volontairement entretenu la confusion ? N'a-t-il réellement pas perçu la distinction entre les deux vies à la lecture du manuscrit? Il est vrai que l'auteur emploie la troisième personne du singulier (il, Godest) pour écrire son autobiographie, ce qui est assez rare dans ce genre littéraire qui privilégie souvent le «je». Il écrit une seule fois que l'histoire du service militaire concerne son frère, puis n'emploie plus ensuite que «Godest», ce qui ne facilite pas la clarté du récit. De plus, la manière de raconter l'histoire donne l'impression d'une restitution d'événements et de sentiments vécus par l'auteur qui, je pense, a fini par se fondre dans le personnage de son frère et s'approprier son histoire.

\section{Les corrections de Jaffrennou}

Cependant, mis à part cette confusion peut-être involontaire de la part de Jaffrennou, et ces choix somme toute nécessaires, d'autres interventions de l'éditeur sur le texte de l'auteur attirent l'attention. Dans la préface au Gwerziou poblus, Jaffrennou se montrait à la fois enthousiaste, mais également immédiatement prudent quant à l'écriture de Godest : "Evit gwir, e rimou n'o deuz ar stum gwiziek o divije bet, mar vije bet Godest eur barz disket : awechou int hir eun tam, awechou na ve ket pell evit trei diwar e vam-skrid, awechou ec'h adlavar ar memez traou, awechou eur gir gallek bennag a zo sklabeet ama hag ahont en e vrezonek... ${ }^{3}$ » (En vérité, ses vers n'ont pas la forme savante qu'ils auraient eue si Godest avait été instruit :

3. Id., p. 12. 
parfois ils sont un peu longs, parfois il se détourne rapidement de son texte orginial, parfois il se répète, parfois il jette ici ou là un mot français dans son breton...).

Jaffrennou légitime de la sorte une intervention de sa part, lui qui sait ce qu'aurait été une écriture plus savante. La comparaison méthodique des passages du manuscrit correspondant aux passages publiés met en lumière un certain nombre de procédés de correction de sa part :

- Au niveau de l'orthographe :

Les choix de correction orthographique de Jaffrennou témoignent d'un curieux rapport à la langue et à l'auteur. Il oscille entre deux tendances qui paraissent contradictoires. D'une part, il corrige Godest afin de normaliser son orthographe qui est approximative et parfois inventive, mélange de formes lues au catéchisme ou dans certains périodiques ou livres très répandus, mais aussi de formes dialectales proches d'une transcription phonétique, comme c'est la pratique chez la plupart des auteurs issus du peuple. Ainsi, Jaffrennou reprend les «divean» de Godest et leur ajoute un «z» ( «divezan»), de même pour les «gwean» qu'il transforme en "goue$z a n »$, certains «eus» sont écrits «ouz», préfère la forme mutée «en eur ziskoe» à "en eur diskoe», et enfin il semi-normalise, sur une sorte de standard carhaisien, les «ari» en «arru», les «dimp» en «'emp».

Mais, d'autre part, cette normalisation se double assez souvent d'une dialectalisation, voire d'une badumisation, alors que Godest avait choisit une forme littéraire. Par exemple, les «aneze» de Godest deviennent «anê», les «deze» «d'ê », les «gouzout» «gout», «loen» «lon», «diragan» devient «dirakan», «goulen» «goul», «kaèz» «kez», et le «dek gwej» fait apparaître le sandhi typique de l'oralité «dek kwech» et le «eta» s'ellipse en «'ta».

Enfin, Jaffrennou va même jusqu'à franciser en «Constantine» l'orthographe du nom propre «Konstantin» que Godest avait établi dans une forme orthographique bretonne.

- Au niveau de la syntaxe :

Sans doute pour gagner en clarté et facilité de lecture, Jaffrennou fait le choix d'ajouter des éléments de ponctuation là où il en 
manque. Comme il coupe dans le corps du texte, il est parfois obligé de réintroduire un nom, un sujet, un objet, afin que lecteur sache de quoi il en retourne et doit aussi introduire des mots de liaison comme «neuze» (alors), «c'hoaz» (encore) pour relier les idées les unes aux autres.

Un ensemble de traits de corrections révèle une recherche de l'éditeur d'alléger le texte de Godest. Il supprime de nombreux «ive» (aussi) et «eta» (donc), petits mots passe-partout, parfois chevilles, parfois porteurs de sens, mais de sens changeant selon les contextes. Comme pour les chevilles des textes médiévaux bretons, c'est lors du passage à une traduction que l'on s'aperçoit qu'ils ne sont pas simples chevilles, mais qu'ils sont difficiles à traduire. Jaffrennou les a-t-il trouvés inutiles ou a-t-il pensé le texte en français ? De même, il supprime un bon nombre de prépositions conjuguées qui apportent pourtant de la précision au texte (gante, aneze, ontan (outañ), c'heman, an eil eus egile...). Il opte aussi parfois pour le style indirect et obtient, à partir de la phrase «...a lavare ennan he c'heunan, gwelet a ran sklaèr a c'han da vean puniset» (il se dit : «Je vois bien que je vais être puni») une phrase plus brève : « gwelet a ree a oa o vont da vean punisset» (il voyait qu'il allait être puni). Il peut changer un groupe infinitif en groupe nominal : "goude bean gret ho c'honje» (après avoir fait leur service) devient "goude ho c'honje» (après leur service). Il peut transformer une proposition relative en groupe verbal ou nominal : «eul lizer, ak a pede anezan da gavet zousi deus he gender» (une lettre qui le priait de prendre soin de son cousin) devient «eul lizer da bedi anean...» (une lettre pour le prier de...) ou "akordi dac'h ar pes a c'houlenet» (vous accorder ce que vous demandez) devient "asanti ganec'h war ho koulen» (donner satisfaction à votre demande). La même motivation est peut-être à l'origine des changements d'un «piou benak a zeuio da lavared» (quiconque viendra dire) en «neb a lavaro» (quiconque dira), d'un «evel ma nefa disket en he vugaleaj» (tel qu'il avait appris dans son enefance) en «...a vihanik» (de l'enfance), d'un «a grie a boués ho fenn» (mot-à-mot : criait de toute leur tête) en "a grie a bouez penn» (mot-à-mot : criait à toute tête), d'un «toukou gant deillou palmes» (des chapeaux en feuilles de palmier) en "tokeier palmez» (des chapeaux en palmiers), d'un «eur vej kroget war ar brezonek» (mot-à-mot : une fois commencé sur le breton) en «eur 
wech krog da vrezonegi» (une fois commencé à bretonner) ou encore de la suppression du double sujet de «sethu godest o tont eur jonj en he ben» (mo-à-mot : voilà Godest à venir une idée dans sa tête) qui devient «ha sethu eur sonj o tond 'n e benn» (et voilà une idée qui lui vient en tête).

L'éditeur passe très souvent de l'imparfait au passé simple que Godest n'emploie pas pour tous les verbes («a lavare» devient «a lavaraz»). Il préfère les phrases à objets post-posés plutôt que de conserver les formules très littéraires et de niveau élevé de Godest avec objets anté-posés : «me da bardon a greis ma c'halon» devient «me bardon d'id», "gant ar boan a neus eus da glevet» devient «ar boan a rez d'ean» par exemple. Il corrige de nombreuses prépositions, transforme de nombreux «evit» en «da» (pour): par exemple, «a oa eur skouer a furnes evit ar iaouankis » devient «d'ar yaouankiz», «evit gouzout» devient «da c'hout». Il préfère, à mon avis fautivement, la préposition «ouzh» à «gant» dans «An daou ael ze, ha me a gomze an eil gant egile». Il supprime la préposition dans "c'henes afat a goms er iez ma kar» pour obtenir «ar iez a gar».

Enfin, Jaffrennou change parfois l'ordre des mots, sans qu'une motivation claire sous-entende ce choix. Par exemple «a lakeas en dorn peb hini aneze, eur pes dek gwennek» (mit dans la main de chacun d'eux une pièce de dix sous) devient «a lakeaz eur pez dek gwennek en dorn pephini anê» (mit une pièce de deux sous dans la main de chaucn), "ha pa oan o tifuni, a oa ar c'hleron o son ar reveill» devient «ha pa oan o tihuni, ar c'hlêron oa o son ar réveil» (lorsque je me réveillai, le clairon sonnait le réveil), «lavaret a rae dezan he c'heunan ar c'homjou man» (il se disait les paroles suivantes) devient «D'ean e-hunan e lavare» (il se disait).

- Au niveau du vocabulaire ${ }^{4}$ :

Une oscillation entre normalisation - autrement dit une tendance à bretonniser le vocabulaire ou à lui donner un niveau plus élevé - et emprunt - autrement dit des propositions de francisation - se retrouve fréquemment dans les corrections de Jaffrennou. Le premier aspect pousse l'éditeur à transformer «asur» en "gwarrant»,

4. Je ne traduis pas les exemples de cette sous-partie car une simple traduction ne permet pas de saisir les différences en jeu. 
«maro dous» en «tremen diboan», «reglet mad» en «reolet», «aprouvi pe annaveout» en «amproui», «net》 en «mik», «èreur» en «nec'hamant», « goulen» en «menad», «brillo» en «luc'hi», «skedi» ou «lugerni», «brillant» en «lufrus» ou «lugernus», "eun transport a joa» en "eur barrad joa», "bras bras meurbet» en «divent», "an oll ornamanchou kaer» en «o dillad», «puniset» en "gwanet», «lijer» en «skanv», «refus anean» en "dic'hrasia anean», «tomder» en «grez», «eskadron zoudardet» en «eskouad», «chadeni » en «erea», «kas» en «ambrouga».

Le deuxième mouvement mène Jaffrennou à abandonner «he zouger monae» pour «e borte-moni», "eun tam bras a arc'hant» pour «eun tamm brao a voni», «eur c'hrok dorn» pour «poignéemain», «breizat iaouank» pour «breton iaouank», «an tok breizat» pour «an tokou Breton», «kiriek» pour «kaoz», «eur pes skorn» pour «eur blokad skorn», "eun de a zikouis» pour «eun devez a repozum», «ober tokou» pour «fabrika tokou», "griet en kis breis izel» pour «kordet e giz Breiz-Izel», «kaset» pour «konduet».

Outre ce va-et-vient entre bretonisation et francisation, on note que Jaffrennou corrige le vocabulaire de Godest en essayant parfois de relever le niveau de langue de l'auteur et parfois en le rabaissant : alors qu'il substitue «amparta» à «muian disket», «kalon Godest a zaouranne» à «Godest a strake he galon», «gwinta» à «gwean», «hopas» à «c'huchas», ou «marvet santelamant» à «marvet en karante doue», il réduit le «madelezus» de Godest à «mad», change un «reas kals a joa» en «reas vad» ou un «komz» en «kaozeal».

Un autre trait remarquable de la correction de Jaffrennou consiste en la suppression systématique d'un des deux éléments constitutifs des doublets synonymiques qu'emploie Godest en grand nombre. Taldir semble ne trouver aucun intérêt à ces doublets et donne l'impression de vouloir simplifier et alléger le texte de l'auteur qui cherche, lui, à gagner en autorité par l'emploi de plusieurs niveaux de langue ou de doublets à visée pédagogique. Certains doublets sont basés sur le principe de la cohabitation d'un mot d'origine bretonne et d'un mot emprunté au français. Jaffrennou, en voulant alléger les doublets, supprime-t-il davantage le mot breton ou le mot français? 
Voici un tableau qui classe les doublets simplifiés à partir de ce critère :

\begin{tabular}{|c|c|}
\hline $\begin{array}{l}\text { Doublets dans lesquels a été conservé } \\
\text { le mot breton }\end{array}$ & $\begin{array}{c}\text { Doublets dans lesquels a été conservé } \\
\text { le mot français }\end{array}$ \\
\hline $\begin{array}{l}\text { eur vedalen (pe eur vedaillen) } \\
\text { tud dirol (pe direglet) } \\
\text { (Estimet ha) meulet } \\
\text { manian (exercie) } \\
\text { ners,( kouraj ha) kalon > nerz-kalon } \\
\text { a defoa boas( pe gustum) > oa boazet } \\
\text { eur strollat (pe eur vandenn vras) } \\
\text { oan (kapabl pe) barek } \\
\text { kaer (ha joaus) meurbet } \\
\text { da (dromplan pe) da douelli } \\
\text { (isplikan, pe) displegan } \\
\text { puri (pe nettaat) he oll dreou } \\
\text { ma dleat (pe dever) a zoudard } \\
\text { (ar merk pe) ar c'hrezen } \\
\text { Sperejou kaer (ha skler) > speredet kaer } \\
\\
\text { skolaj ar bourkou, (selon l'école } \\
\text { primaire). > skol ar bourkou } \\
\text { en eisvet ruzumant houzardet (pe } \\
\text { en galek au huitième (regiment des } \\
\text { hussards.)); } \\
\text { ar c'hrok dorn (poignet main) } \\
\text { eur feunteun skornet, (c'hanvet en gallak } \\
\text { (la fontaine glacial,)) }\end{array}$ & $\begin{array}{l}\text { (koant ha) faro > faro } \\
\text { Sperejou zimp (ha dister) > speredet simpl } \\
\text { ce jeune soldat aprit très bien (da lavaret eo, } \\
\text { ar zoudard iaouank man a zik meurbet mad) } \\
>\text { ce jeune soldat a appris très bien } \\
\text { dre (he c'halout ak) he volonte } \\
\text { treo vil (ha c'hudur) } \\
\text { eur postur (pe en eur stum) } \\
\text { en koler (pe en buaneges) } \\
\text { plas, (pe lec'h) eur zoudard } \\
\text { da ordrenans, (pe da vevel) } \\
\text { eur woez horup, (pe eur woez spontus) }\end{array}$ \\
\hline
\end{tabular}

Sur 29 doublets relevés, les $2 / 3$ mettent en avant un mot breton contre $1 / 3$ qui donne la préférence au mot d'origine française. La position de l'éditeur, bien que donnant nettement plus de place aux mots bretons, ne semble toutefois pas radicale en la matière. De plus, on peut noter que Jaffrennou tient au critère esthétique et qu'il préfère l'alternance d'un mot breton et d'un mot français plutôt que le tout breton. Par exemple, il choisit dans un premier temps «oc'hazeuli (pe adori)» pour «adorer», puis deux lignes en dessous «o c'hadori (pe azeuli)». Cette même pratique se retrouve d'ailleurs à partir

5. Alors qu'il a par ailleurs corrigé le mot breton en «poignée-main». 
des doublets de type dialectaux : il emploie d'abord, pour «matin», «ar mintin, (pe ar beure)» et quelques lignes plus loin «(mintin pe) beure».

Les autres doublets synonymiques regroupent deux termes bretons et les motivations de l'éditeur quant à la suppression de l'un des termes n'est pas évidente. Pour les doublets dialectaux, Jaffrennou ne conserve qu'une forme: (na ouzes pe) na oares; an abarde, (pe ar pardaes) ; raktal,( pe dustu) ; ober (kerniou, pe) korniou da vutunet $>$ korniou butun). Pour les autres, il semblerait qu'il ait cherché soit à éviter les termes trop forts, trop excessifs (an hegas (pe ar brezel), humb (a joaûs), méz (ha keun) dean, furnes (ha lealdet), (ornet ha) kurunet, an oll enor (ak an oll gloar), Mes allas ar re vras, (pe mar ententet gwelloc'h, an dud disket bras, pe tud a gargou) pe tud pinfidik > ar Re Vraz, ar Re Binvidik) ou à privilégier des termes plus rares ou recherchés (diouganet a neus (ha laret), an drouk ele (pe an diaoulou), neuze a oant galvet (pe c'hanvet), an oll dreo (a vije) gourc'hemenet, pe lavaret dean ober gant he vestrou), ma fedenou ordinal, (pe evel ma oan kustum da ober), siret (pe torchet)).

- Au niveau du contenu :

Il n'est pas possible ici d'être exhaustif, d'autant plus que les interventions sur le contenu du texte de Godest vont d'un changement de mot (comme par exemple «evel diou askel eur vran o nijal» (comme les ailes d'un corbeau en vol) qui devient «evel diou askel eur sparfel o nijal» (comme les ailes d'un épervier en vol), ou encore, pour «chant», un «gwerz» changé en «soniou») à la suppression de très longs passages de plusieurs pages. Je ne mettrai ici en avant que les grandes tendances relevées dans les interventions de Jaffrennou sur le contenu de l'autobiographie en question.

Il apparaît que Jaffrennou modifie le sens du texte principalement par suppression et non par ajout ou modification du sens des phrases. Quelques transformations trahissent un souci d'ancrer le propos dans un cadre breton : ainsi passe-t-il d'un «breis izel» (Basse-Bretagne») à un «Breiz» (Bretagne»), d'un «tam kanaouen neve» («une nouvelle chanson») à un «tamm kanaouen vrezonek» («une chanson bretonne»), d'un «gwir zoudard a vreis izel» («un vrai soldat bas-breton») à un «bugel da Vreiz-Izel» («enfant de Basse-Bretagne»). 
La très grande majorité des interventions de Jaffrennou consiste donc en des suppressions. Celles-ci révèlent à la fois le souci esthétique et littéraire de l'éditeur, mais indiquent également ce qu'il considère thématiquement ou discursivement comme superflu, inutile, voire dérangeant pour le lecteur. Il se trouve en fait que Jaffrennou considère Godest comme maladroit ou excessif dans plusieurs domaines :

Premièrement, c'est une esthétique de la concision que souhaite transmettre Jaffrennou, esthétique à l'opposé de celle mise en place par l'auteur qui, au contraire, consolide l'ancrage de son personnage dans le réel par force détails, consolide sa propre position d'auteur par une rhétorique basée sur des phrases longues et reformulées. Les redites ou phrases se rapprochant trop d'une expression qui vient d'être formulée sont ainsi supprimées par l'éditeur pour des raisons de style. Comme pour les doublets, Jaffrennou ne conserve qu'une formulation, considérant sans doute les reformulations comme des lourdeurs d'expression. De la même manière, il n'hésite pas à couper dans les énumérations qu'il considère comme alourdissant le propos, comme par exemple lorsque Godest énumère les ordres qui sont donnés aux soldats à l'armée : "par le flan droit, par le flan gauche, (tête droit, tête gauche, demi tour, a droit, marché de pied gauche) $[\mathrm{sic}]^{6} »$.

Deuxièmement, Jaffrennou défait l'ensemble du cadre dans lequel Godest a voulu insérer son propos, à savoir un cadre qui donne la priorité à la religion et à la morale. En ôtant ce cadre rigide, l'éditeur pense sans doute libérer le contenu, les événements et anecdotes racontés, et ainsi se rapprocher du réel. Or Godest a souhaité déréaliser son propos pour le sublimer et sublimer le héros du texte, c'est-à-dire lui-même. Rien de tel que les alexandrins pour faire de Jean Conan un acteur de tragédie classique, rien de tel qu'une protection sans cesse réitérée de Dieu pour faire de Julien Godest un élu.

Godest ouvre son autobiographie par «En hanou an tad, ak ar mab, ak ar speret zantel, evelse bezet gret. Doue a rok, ha me warlerc' $h$ » («Au nom du Père, du Fils, du Saint Esprit, ainsi soit-il. Dieu

6. Je reproduis le texte dans l'orthographe (fautive) de l'auteur. 
avant, moi ensuite») et ceci donne toute une orientation à son texte, orientation que ne reprend pas l'éditeur qui supprime également presque systématiquement les remerciements à la Vierge, aux saints et aux anges pour ne conserver que le remerciement à Dieu, quand il ne le supprime pas également : "trugarekat a ran doue, (ar verc'hes ar zent ak an ele)» («Je remercie Dieu, (la Vierge, les saints et les anges »)). Jaffrennou ampute également les prières du personnage, quand il ne les supprime pas tout simplement, comme par exemple: «ma doue ma c'hrouer me ro dac'h ma c'halon ma c'horf ha ma ine, kement tra a rin en deves man a betek fin ma bue, (gret din ar c'hras me ho ped, na rin nep drouk, ha gret ma rin peb tra mad, evit ho kloar ha zilvidiges ma ine, en goulen a ran dre meritou jezus krist ma zalver)» («Mon Dieu, mon Créateur, je Vous donne mon cœur, mon corps et mon âme, tout ce que je ferai ce jour et jusqu'à la fin de ma vie, (Faites-moi la grâce, je Vous prie, que je ne commette aucun mal, et Faites que je ne fasse que le bien, pour Votre gloire et le salut de mon âme, je Vous le demande par les mérites de Jésus Christ mon sauveur). Il se passe de fort nombreux passages dans lesquels l'auteur loue Dieu, comme par exemple : «Eur wieges oll galoudek ha dispar, a neus an aoutrou doue, evit ober peb tra en eur fesson ken burzudus, rak dre ar voien ze, a ro lec'h dan oll dud da vean hûmp ha d'ho c'hars da zisprizout an dud all, / Rei a ra ive an aoutrou doue dre ar voien ze, eun anaoudeges sklèr dan oll dud, da anaout eo doue oll galoudek, oll karantezus, ak oll madelezus en kever an oll» (Dieu a une connaissance toute-puissante et extraordinaire pour faire chaque chose d'une manière si merveilleuse car, ainsi, il donne à tous la possibilité d'être humble et les empêche de mépriser les autres. / Dieu fait également ainsi clairement savoir à tous les hommes qu'Il est Dieu tout-puissant, tout-aimant et tout-bienfaisant envers tout le monde»). Il polit l'image de Godest en lissant les côtés excessifs de son exaltation religieuse.

Ce cadre religieux omniprésent se traduit également chez Godest par un souci permanent de faire des vies racontées dans son texte des prétextes à propager la bonne parole, une morale catholique martelée. Le souci pédagogico-moralisateur se glisse à tout moment de la narration, l'auteur faisant des pauses qu'il nomme «Kelennadurez» (leçon) et prend la distance et la hauteur du prêtre en chaire pour tirer leçon de l'exemple : "C'hoant a meus da rei kelenadures dan 
dud war disobeisans pe dizentidiges adam ak eva, an dizentidiges a zo eur gwal bec'het » (Je veux faire une leçon aux gens sur la désobéissance d'Adam et Ève, la désobéissance est un grand péché»), «Evit isplikan mad an treou ze, eo ret din trei anezou diwar trajedien Godest, pe diwar eun darn deus an treou, a neus tremenet en he vue, evit kelen an dud war ar gwir furnes, kristen, ha da chom en ho bro, ha da zerc'hel stard dar brezonek», "C'heuillomp breman adare, tradgedien Godest, lezet a goste aboue an numero 107 evit kelen pobl breis izel da garet ho bro, ha da chom enni, da vevan en kristenien vad ha fur, C'heuillomp c'hoas trajedien Godest, da c'hortoz kavet c'hoas eur pleg mad da gelen an dud...» (Pour bien expliquer cela, je dois faire une digression par rapport à la tragédie de Godest ou à certains événements de sa vie, afin d'enseigner aux gens la vraie sagesse chrétienne, la nécessité de rester au pays et de garder fermement le breton). Jaffrennou gomme la presque totalité de ces pauses de prédication.

Troisièmement, Jaffrennou a voulu atténuer la position nettement patriotique bas-bretonne de Godest en supprimant de nombreux passages où l'auteur fait mention de son attachement à son pays, de sa fierté à être Breton, ou de la supériorité des Bretons sur les autres peuples. Les lecteurs ne lisent donc pas la pensée qui traverse l'esprit du frère de Godest lorsqu'il sent qu'il va sans doute être puni par un colonel qui ne croit pas au rêve fait par Godest qui affirme avoir été au paradis : «Eur gwir vreizat na dle ket spontan rok he skeud, ret eo din eta kemer kalon eur gwir vreizat, ha lavaret, breizat a c'hon, breizat a vin, ha dan nep em c'hlasko me en asur erfad, me a ziskoue dean a c'hon eur gwir vreizat» (Un vrai Breton ne doit pas avoir peur de son ombre, il me faut donc avoir le cœur d'un vrai Breton et dire «Je suis Breton et le resterai» et essayer de montrer parfaitement à tous que je suis un vrai Breton). Jaffrennou fait des coupes dans l'extrait qui relate les sauts effectués par Godest au-dessus de plusieurs chevaux, fait relaté comme un exploit qui provoque l'admiration des autres soldats: "n'oc'heus ket ezom da c'houlen, c'henes a zo eur breizat, neuze a lavarent, (o, lala), quel (brave) zoldat, (o pegen gwiek eo ar vretonet (ils sonts sans pareille) na man ket ho far war an douar) » (Il n'est pas nécessaire de le demander, c'est un Breton. Alors ils dirent: «(Oh lala), quel (brave) soldat! (Comme les 
Bretons sont intelligents, ils sont sans pareille, il n'y en a pas d'autres sur terre à leur ressembler !)). Enfin, il condense à l'extrême la comparaison que fait Godest entre la Bretagne et Paris :

«ma bro gaer breis (izel, bro en lec'h ma ve ket a vrezel,) bro ar zioulder ak ar peuc'h, (bro an ners) ak ar iec'het, (bro an dud joaûs ha laouen,) bro ar furnes (ha kalonek, bro an unvaniez ak an oberou mad, bro en lec'h ma tiwan bemde, magadures evit an dud ak evit ar loenet, bro en lec'h ma eo aez dan dud bevan mar karent poanial, bro en lec'h ma zo bara nerzus ak efaj ak efaj ar vro a zo iac'hus, bro en pehini en neve amzer zo kaeroc'h evit na eo jardin ar plant en Paris, rak an treo a veler en jardin plant en Paris, a zo treo digaset dar lec'h ze gant an dud, treo ak a ia buan da netra, mes en breis izel dan neve amzer, a veler peb tra ac'hat nevei pe neveat, evel en he gerder kentan.

Ar goukouk, ak ar gwenidel a zeu er vro da zisklerian an neve amzer, an oll jardinou a zeu peb tra enne da neveat, an oll douarou a zeu peb tra da neveat enne, dre c'halout an doue oll galoudek, a neus roet he natur da bep tra evit kemense, ha peb tra oll zent c'herve he natur eus an eurs roet dezan gant doue, c'hep gwej abet dizenti an disteran tam,) en lec'h aman en Paris na neus nemet bue fall, ha dizentidiges traitouraj (ha revolt, ak erfin) ar maro ar muan kri (goneet ganimp, dre hon gwall vue, on disurjou, ak hon disprizans en kever doue, hon c'hrouer ak hon mad obereour karantezus).

Mon beau pays la (Basse-)Bretagne, le pays du calme et de la paix, (le pays de la force) et de la santé, (pays des gens joyeux), pays de la sagesse (et du courage, pays de l'unité et des bonnes actions, pays où germe quotidiennement la nourriture pour la population et les bêtes, pays où il est facile de vivre si l'on aime la travail, pays où il y a du pays nourrissant et de la boisson et la boisson de ce pays est bonne pour la santé, pays où le printemps est plus beau que le Jardin des Plantes à Paris, car les plantes que l'on voit au Jardin des Plantes à Paris sont des plantes rapportées par les hommes et qui ne tiennent pas longtemps, alors qu'on Basse-Bretagne, au printemps, les nouvelles pousses arrivent comme dans leur première beauté.

Le coucou et les hirondelles arrivent au pays pour annoncer le printemps, dans tous les jardins pointent les nouvelles pousses, tout germe dans les champs, par la force du Dieu tout-puissant qui a pour cela donné sa nature à toute chose, chaque chose obéissant selon sa nature à l'odre qui lui a été donné par Dieu, 
sans jamais désobéir en rien), alors qu'à Paris il n'y a que mauvaise vie, désobéissance, traitrise, (et révolte et finalement) la mort la plus cruelle (bien méritée à cause de notre mauvaise vie, nos désordres et notre mérpris vis-à-vis de Dieu, notre créateur et notre charitable bienfaiteur.)»

Quatrièmement, le toilettage du texte de Godest passe par un rabotage de la presque totalité des passages caractérisés par la vantardise du narrateur. Jaffrennou a pu estimer que ce manque d'humilité, soit allait à l'encontre des préceptes religieux, soit donnait une place trop importante au personnage qui effaçait par là les événements. Rien ou presque n'apparaît donc des «Godest a oa ken c'hoantek da ziski ha ken sklèr a speret» (Godest était si volontaire pour apprendre et si clair d'esprit), des «ne lavare gwej abet komjou lous ha c'hudur» (il ne disait jamais un seul mot grossier ou obscène), «dre ma oa karet gant an oll zoudardet, ha gant ar mestrou, na vije gwej ebet gwal vec'hiet a labour, da vianan na vije lavaret dean ober netra c'hep na vije dleet dean en ober» (comme il était aimé de tous les soldats et des maîtres, il n'était jamais trop chargé de travail, ou du moins on ne lui demandait rien de plus que ce qu'il devait faire), «na éfé gwej abet da vean meo, na memes da droubli he anoudeges vad, bean humbl, zentus ak obeisant ha gant eun aèr kontant ha joaùs» (il ne buvait jamais pour se soûler, ni même jusqu'à troubler sa conscience ; être humble, obéissant et l'air joyeux), «sel aze pegen kalonek den eo c'henes, c'henes a ro enor d'he vro, c'henes a zo eur gwir vreizat, na veler ket he bar» (regarde comme il est un homme courageux, il fait honneur à son pays, c'est un vrai Breton, il est extraordinaire), ou encore des "Godest a vije roet dean enor abalamour da ze, mes Godest na gemerre ket a lorc'h evit an dra ze, bean a oa bepret ken humbl ha kent, mes rei a rae ar gloar a gemense da zoue, rak Godest oare mad penoz a oa doue, ha doue c'hepken, a nefa roet dean, an ners, ar gouraj, ak an ners kalon, ak an oll dalanchou mad evit ober an oll dreou ze, ken zouezus da zonzal enne» (On faisait honneur à Godest pour cela, mais Godest ne s'en enorgueillait pas pour autant. Il était toujours humble, mais il donait à Dieu la gloire de tout cela car Godest savait bien que c'était Dieu, et Dieu seulement, qui lui avait donné la force le courage et tous les bons talents pour réaliser tout ces choses si surprenantes à 
imaginer). Jaffrennou gomme également les «bravo» que les autres soldats, admiratifs de la conduite de Godest, lui lancent régulièrement en frappant dans les mains.

L'objectif de Godest étant de tirer leçon de sa propre vie, de montrer en quoi sa vie est faite de comportements et sentiments exemplaires pour ses concitoyens, et Jaffrennou ayant souhaité retirer ce cadre pédagogico-moralisateur et les excès de vantardise de son protégé, presque toutes les mentions de l'exemplarité de Godest disparaissent du texte publié : par exemple, plus de «Godest eo gwir skouer vad an arme» (Godest est le vrai exemple de l'armée), «Sethu aze pegen talfoudus eo ar skouer vad gret eveltan» (voilà comme est important un bon exemple comme lui), «Ar zoudarded all deuet da vean reglet mad dre ar skoer vad a C'hodest» (les autres soldats finirent par être bien réglés sur le bon exemple de Godest), Godest «a teuas da vezan skoer an oll vevelien, ha memes skoer an oll dud a renk izel. Ha meur a c'hini a gemeras he skouer en he fesson da vevan, kement den ha anaveé a roe meulodi dezan, abalamour d'he vue vad, ak abalamour dar skouer vad a roe dan oll» (Godest finit par devenir l'exemple de tous les valets et même l'exemple de tous les gens de basse condition. Et plusieurs prirent exemple sur sa manière de vivre. Tous ceux qui le connaissaient le louait pour sa bonne vie et pour le bon exemple qu'il donnait à tous). Ou «Me a bed eta an oll zoudardet, ak ive an oll dud a bep renk, a bep oad, a bep stad, ak a bep micher, da len pe da jilao len trajedien Godest, pe eun darn deus an treou a dremenas en he vue, ha me ho ped oll da gemer skouer diwar bue Godest» (Je prie donc tous les soldats et aussi tous les gens de toute classe, de tout âge, de toute condition, et tout métier, je prie tout le monde de prendre exemple sur la vie de Godest).

Enfin, cinquièmement, Jaffrenou laisse de côté quelques éléments sous la plume de Godest qui pourraient lui avoir semblé politiquement ou éthiquement assez peu corrects, notamment concernant l'image de l'armée. Il a préféré modérer les propos excessifs dans la bouche des gradés de l'armée : «penoz (pen) breizat louz, te a da oll vredeur (koz) vreizis (diskiant)» (comment, (espèce de) sale Breton, toi et tous tes frères (de sales) Bretons (incultes)) ; modérer les comparaisons ou qualificatifs affublés aux chefs ou aux activités des soldats contre la Commune : selon Jaffrennou le colonel 
se contente de «dire», alors qu'avec Godest «a lavaras evel eun tirant» (dit comme un tyran); les soldats obéissants de Jaffrennou étaient en fait des soldats révoltés chez Godest ; les insurgés de la Commune se contentent de se défendre sous la plume correctrice de Jaffrennou alors qu'ils se défendaient «evel tud konnaret» (comme des gens enragés) d'après Godest. Jaffrennou fait disparaître la moitié des sévices subis par certains soldats à l'armée : "disprizet goapet, (gret dean dispign he arc'hant, gret dean bourdou en peb kis, diskaret he wele, c'harzet da gousket,) gret dean ober labour ar reo all ak he re he c'heunan » (méprisé, moqué, (à qui on a fait dépenser tout son argent, à qui on a fait des piques en tout genre, défait son lit, empêché de dormir), à qui on a fait faire le travail des autres en plus du sien). Alors que Godest rendait la cruauté de la situation lors des massacres sous la Commune par une énumération qui exprime l'empêtrement dans lequel le narrateur se trouvait ( $a$ tol he dreit, gwej war ar c'horfou maro, gwej o lampat dreiste, gwejou all a kouee en he c'hourve, o klask c'hars da lakat he dreit warne» (il marchait parfois sur les corps morts, parfois sautait au-dessus d'eux, parfois il tombait de tout son long, cherchant à prendre appui sur eux), Jaffrenou la réduit considérablement par un simple «tol a ra e dreid war gorfou maro astennet» (il marchait sur les corps morts allongés).

Dans la même perspective du souci d'expurger le texte de propos peu valorisants, Jaffrennou balaye l'idée d'action pouvant se faire sans loi ("pe oa lezen da ober pe na oa ket, gret a oa bepret, meur a dra ve gret c'hep lessen» (qu'il y ait une loi pas pas, on le faisait. On fait beaucoup de choses sans loi)), l'idée que la petitesse est perçue comme un défaut («Kouskoude ar plac'h man na oa ket deus ar merc'het bras, na oa ket ive bian bian, eun draik benak bianoc'h evit ar vent komun» (pourtant cette fille n'était pas du lot des filles de grande taille, elle n'était pas non plus très petite, juste un peu plus petite que la taille habituelle) devient «Eur plac' $h$ a vent vihan a oa hi» (elle était de petite taille), et l'idée que l'hérédité en matière de comportement n'existe pas : «diwar tud kapabl, a teu tud n'int ket kapabl / ha diwar tud disleal, a teu tud leal /diwar tud traitour a teu tud evel ma eo dleet, ha diwar tud zot, a teu tud fur / darn all, a sten d'ho c'herent eun tamik, ha kouskoude int kals disc'henvel onte» (des gens travailleurs ne donnent pas forcément 
naissance à des gens travailleurs, et des gens déloyaux donnent des gens loyaux, des traitres donnent des gens comme il faut, et des sots donnent des sages; d'autres tiennent un peu de leurs parents et tout en étant pourtant différents d'eux).

La comparaison entre le manuscrit de Godest et les extraits publiés par Jaffrenou met en lumière plusieurs aspects de l'intervention de l'éditeur :

- le souci de bretonniser et normaliser le texte, tant dans sa forme que dans son contenu, mais de manière modérée, voire en réintroduisant de la francisation ou de la badumisation si l'auteur s'est adonné à trop d'auto-correction par rapport à son breton local, ceci afin de ne pas donner l'impression de retouches trop importantes, de conserver ou donner une part d'authenticité, la couleur «populaire» du texte, plus «naturelle», plus «locale».

- le souci de contenir le texte en gommant tout ce qui a été perçu comme excessif chez l'auteur-narrateur-héros, tant la rigidité du cadre dans lequel s'insère le discours, tant dans les débordements discursifs par rapport à un cadre imaginaire de bonne pensée de Jaffrennou.

- le souci d'esthétisation qui entre parfois en concurrence avec le premier énoncé. Il s'agit de faire beau, court, concis, en bref, répondre à un cahier des charges répondant aux attentes supposées d'un lectorat.

\section{Le néo-néo-paternalisme bourgeois de l'Emsao}

\section{Une image embourgeoisée du peuple}

Le long manuscrit de Julien Godest est fabriqué d'un ensemble de phrases abordant divers thèmes, écrites dans une certaine esthétique et rendues dans une certaine forme linguistique, et ce texte étant une autobiographie, il dit plus que jamais la personnalité d'un auteur, la vie d'un homme du peuple telle qu'il a souhaité qu'elle passe à la postérité. La publication de François Jaffrennou témoigne du carrefour où se trouve l'Emsao au début du $\mathrm{XX}^{\mathrm{e}}$ siècle : prendre appui sur le peuple, mais en transmettre une image polie. 
Jaffrennou a été frappé par la personnalité hors du commun de Godest et a voulu à tout prix le faire connaître en le publiant, mais il n'a finalement pas cherché à comprendre sa personnalité. Il a porté un regard sur le texte qui relève du jugement de valeur et révèle sa propre vision du peuple. Son regard a perçu à certains endroits un cadre trop étroit et à d'autres une absence de cadrage, alors que c'est par ces outils-là que l'auteur cherchait à se sublimer. Jaffrennou a trouvé Godest pas assez libéré du cadre catholique et de l'intention moralisatrice, et trop excessif, hors de la bienséance et de la prudence en matière de patriotisme, de foi, de mise en valeur de soi. Il a ainsi reformé un cadre différent de celui de Godest et correspondant aux règles de la classe bourgeoise à laquelle il appartient : patriote mais pas obsédé, catholique mais pas fanatique, conduite exemplaire mais rapport mesuré aux autres.

L'image embourgeoisée de Godest que Jaffrennou tire de celle fabriquée par l'auteur lui-même lui permet de montrer que le peuple compte pour la nouvelle génération de l'Emsao, que la classe bourgeoise peut jouer un rôle d'aide, que le peuple peut être conscientisé et intégrer le mouvement breton ${ }^{7}$, que l'union entre le peuple et la bourgeoisie de l'Emsao est possible. C'est dans ce sens que va la proximité exprimée dans $\mathrm{Ar} B o b l$ entre le peuple et la bouregoisie qui se dit elle-même issue du peuple, tout comme l'intrigue de la pièce de théâtre de Jaffrennou Ar Bourc'hiz lorc'hus qui traite du rôle de la bourgeoisie aux côtés des paysans par l'intermédiaire d'un bourgeois, nommé Aotrou Teo, et qui, d'un homme bourru, vivant dans l'opulence et l'aveuglement («teo» signifiant «gros» en breton) devient clairvoyant et se rallie à la cause de la terre, de la sincérité et de l'amour, c'est-à-dire se glisse dans le rôle qu'il a désormais à jouer, le nouveau dieu de la société basbretonne («Theo» signifiant «Dieu» en grec).

La bourgeoisie de l'Emsao prend le relais de la noblesse régionaliste et de son paternalisme. Elle écarte la noblesse et prend sa place, sans rompre véritablement avec le fond idéologique du soin et de l'accompagnement du peuple. C'est ainsi que Jaffrennou justifie

7. Publicité pour les Gwerziou poblus de Godest, par Le Liseur : «quelle belle réponse à ceux-là qui mentent sciemmement, en écrivant que le peuple se tient en dehors du mouvement breton!» 
la nouvelle place de la bourgeoisie conscientisée auprès des paysans et des ouvriers bretons : elle «sait ce que sont leurs besoins, leurs aspirations, ce qu'est leur vie, et elle fera tout son possible pour leur montrer le chemin qu'il serait bon d'emprunter pour vivre dans le bonheur, ensemble, en améliorant leur condition à eux et celle de leur race $^{8} \gg$.

\section{"Si le peuple ne convient pas, changez de peuple» (Brecht)}

Jaffrennou symbolise cette étape intermédiaire de l'Emsao qui se détache progressivement des modèles bretonistes, des nobles régionalistes, pour se diriger vers plus d'autonomisme, puis vers le nationalisme, mais qui ne méprise pas encore le peuple. En donnant une place à l'expression populaire, il donne au peuple une certaine autonomie d'existence, et en lui offrant de la nourriture intellectuelle, il le considère comme digne d'épanouissement, ce que ne faisaient pas les générations précédentes. Par ailleurs, il fait là ce que ne feront plus les hommes de la génération suivante qui, dès 1925 , dans Gwalarn, tournent le dos au peuple en méprisant sa langue et sa littérature, et en inversant les flux de valeurs : le peuple ne sera alors plus source de valeur linguistique et littéraire, c'est l'élite intellectuelle qui sera en charge de lui apprendre ce qu'il doit être.

Toutefois, aucune étape dans l'histoire de la littérature de langue bretonne ne témoigne d'un intérêt pour le peuple tel qu'il est. Les premiers regards tournés vers le peuple au début du XIX ${ }^{\mathrm{e}}$ siècle se font vers un peuple rêvé et idéalisé par les auteurs et les premiers collecteurs. La Pangée des auteurs gwalanistes ${ }^{9}$ éloigne d'une autre manière, mais tout autant, sinon davantage, de la réalité. Jaffrennou et les hommes de cette génération intermédiaire n'échappent pas à ce phénomène qui fait aux auteurs ou éditeurs se prendre les pieds dans le nœud de la rencontre entre le peuple rêvé dans sa pureté et la réalité. Par exemple, la non-scolarisation de Godest en fait un auteur «pur» selon les éditeurs, mais cette même non-scolarisation entraîne nombre de maladresses dans son écriture, ce que prouvent les nombreuses corrections mises en avant dans cet exposé. On rêve

8. «Petra omp ?», Ar Bobl, n 1, Carhaix, 24 septembre 1904.

9. Voir Ronan CAlvez, La Radio en langue bretonne. Roparz Hemon et PierreJakez Hélias : deux rêves de la Bretagne, Rennes, PUR-CRBC, 2000. 
donc d'une pureté, loin de Paris, loin de la loi, loin des révoltes et de la haine, et on pense plutôt à ce "vrai» peuple «ha dre ze e klevomp ar bobl vreizad, ar bobl honest ha mad en he c'halon, ar bobl kristen ha feal da gement tra gaer a zo en ho c'horn douar a Vreiz» (et par cela nous voulons dire le peuple breton, le peuple honnête et bon de cœur, le peuple chrétien et fidèle à toute belle chose se trouvant dans notre bout de terre de Bretagne) ${ }^{10}$.

10. «Petra omp ?», art. cité. 
